\title{
Guest editors' introduction to the special issue on digital libraries for musicology
}

\author{
Kevin R. Page ${ }^{1}$. J. Stephen Downie ${ }^{2}$
}

Published online: 23 February 2019

(c) Springer-Verlag GmbH Germany, part of Springer Nature 2019

Many digital libraries have long offered facilities to provide multimedia content, including music. However, there is now an ever more urgent need to specifically support the distinct multiple forms of music, the links between them, and the surrounding scholarly context, as required by the transformed and extended computational methods being applied to musicology and the wider digital humanities. These challenges are being met by the next generation of music digital library systems, their application and use in musicology, technologies for enhanced access and organization of musics in digital libraries, bibliographic and metadata for music, intersections with music linked data, and the challenges of working with the multiple representations of music across large-scale digital collections

Dissemination of the interdisciplinary work at the intersection between digital library and musicological research has coalesced and grown around the international Digital Libraries for Musicology (DLfM) conference, ${ }^{1}$ growing out of a workshop series begun at the special conjoined Digital Libraries conference convened at London in September 2014. The eight articles in this special issue report upon representative topics, undertaken by key researchers, from this emerging international community. Authors hail from the following institutions: Birmingham City University, UK; British Broadcasting Corporation, UK; Columbia University, New York, USA; Dundalk Institute of Technology, Ireland; Goldsmiths, University of London, UK; Lancaster University, UK; Meertens Instituut, Netherlands; National University of Ireland, Ireland; Royal College of Music, UK;

\footnotetext{
${ }^{1}$ https://dlfm.web.ox.ac.uk/.

$凶$ J. Stephen Downie

jdownie@illinois.edu

Kevin R. Page

kevin.page@oerc.ox.ac.uk

1 Department of Engineering Science, Oxford e-Research Centre, University of Oxford, Oxford, UK

2 School of Information Sciences, University of Illinois at Urbana-Champaign, Champaign, USA
}

Samsung, Inc., UK; The Open University, UK; University of Alicante, Spain; University of Birmingham, UK; University of Oxford, UK; Répertoire International des Sources Musicales offices in both Germany and Switzerland; Utrecht University, Netherlands.

The papers assembled here each combine one or more real-world musicological motivations with one or more innovative digital library technologies or techniques. Motivations include the desire to more closely study performance and listening practices, to improve the use and reuse of multifaceted music-related resources, to better represent structural analyses, and to provide scholarly access to annotations found on scores. Technologies and techniques explored include crowdsourcing, machine learning, semantic encodings, semantic alignment, and linked data.

The first paper, "Applications of RISM Data in Digital Libraries and Digital Musicology," describes the Répertoire International des Sources Musicales, a significant and large international index of manuscripts and printed music. The authors set out detailed descriptions of the growing quantities and properties of RISM data and its applications, where this is supporting digital music research, and how projects are using — and might feed back—RISM data. The inclusion of searchable music incipits within the RISM OPAC provides a particularly compelling example where music encodings (in this case Plaine and Easie Code) can bring additional, music specific, features to digital libraries.

"Documenting a Song Culture: The Dutch Song Database as a Resource for Musicological Research" describes a thematically focused digital corpus containing more than 173,000 references to song occurrences in the Dutch and Flemish language, from the Middle Ages up to the present, as well as over 18,000 descriptions of song sources, such as song books, manuscripts, and recordings. The Dutch Song Database is long-running project, pioneering in many ways, initiated by a musicologist and which has informed and influenced numerous studies sets out in the paper. The authors describe the technical design of the database, including how diverse musical sources are linked, documenting valuable 
experiences and insights for the development of resources of this type.

The third paper, "On Providing Semantic Alignment and Unified Access to Music Library Metadata," explores solutions for reconciling entities held across digital music libraries. The authors implement and evaluate SALT, the Semantic Alignment and Linking Tool, which provides an interface for musicologists to assert co-references of crosscorpora concepts. Given metadata in RDF format, SALT uses two mechanisms to present candidate matches to the user: string similarity and 'semantic context', the latter using emergent graph structures to identify equivalent, but structurally distinct, contextual patterns within the datasets. The authors apply their tool to link digitized scores in the British Library with broadcast data from the BBC.

"Heuristic and Supervised Approaches to Handwritten Annotation Extraction for Musical Score Images" reports on experiments to extract handwritten notes made on music scores. Such annotations are of musicological significance because they reveal the practices and thinking of those using the scores, in this case conductors. The authors apply machine-learning techniques to digital facsimiles of the scores, firstly evaluating and refining a heuristic process for isolating the annotations into a separate layer, and then developing a pixel-based supervised classification technique, using the output of their earlier process as the basis for creating a manually corrected training set.

The paper "Crowdsourcing Linked Data on Listening Experiences through Reuse and Enhancement of Library Data" addresses difficulties stemming from the paucity of high-quality structured information that captures usergenerated music listening experiences. The lack of data in this area has hindered research progress in the domain of musical perception. To overcome this serious impediment to scholarship, the authors developed and deployed the Listening Experience Database (LED). The LED, which was created via crowdsourcing, contains some 10,000 records, and also incorporates and reuses open data from the British Library and MusicBrainz using Linked Data structures.

The sixth paper, "Encoding Music Performance Data in Humdrum and MEI," focuses on a specific area of musicology, the study of performances, and sets out the motivation and implementations for digitally capturing perceptually relevant descriptions of the performance in conjunction with symbolic score encodings. Having surveyed the current practice and needs of music scholars, the authors identify the necessary note-level, beat-level, and continuous performance concepts necessary to realize an sufficient encoding. This is them implemented as extensions to Humdrum and the Music Encoding Initiative (MEI).

Many musicological studies investigate the nested structures found in music. Presenting the results of such studies in way that takes advantage of modern digital library capabilities has been problematic. To remediate this issue, the authors of "An MEI-based Standard Encoding for Hierarchical Music Analyses" present a proposed solution that builds upon both ME and Text Encoding Initiative (TEI) elements to construct a sophisticated representation that expresses both semantic and non-semantic information. By bringing together these elements, the new encoding allows musicologists to express both the formal aspects of their analyses while simultaneously revealing the graph or tree structures they want the reader to see.

The eighth and final paper in this collection, "Curating and Annotating a Collection of Traditional Irish flute Recordings to Facilitate Stylistic Analysis," describes the creation and potential uses of an exciting new ethnomusicological resource designed to support musicologists studying cultural change, musical heritage, and music making. In addition to outlining the technical and statistical aspects of their collection, the authors also highlight seminal recordings of key Irish flute music performance and provide insightful analyses concerning the interplay of performance style and geographic context.

In this special issue, we can see how advances in one domain facilitate advances in the other. The needs of musicology inspire digital libraries to develop new approaches to data encoding, handling, and access. Digital libraries offer musicology novel mechanisms for scholars to combine, reuse, and publish their resources and scholarship. As one reads through the papers, it becomes readily apparent how much the digital library and musicology worlds can — and do-benefit from interacting.

Publisher's Note Springer Nature remains neutral with regard to jurisdictional claims in published maps and institutional affiliations. 